\title{
A SIMPLE AND FAST RP-UHPLC-PDA METHOD FOR DETERMINATION OF ISRADIPINE FROM POLYMERIC NANOCAPSULES
}

\author{
FERNANDA MALAQUIAS BARBOZA, GUILHERME DO ANJOS CAMARGO, AMANDA MARTINEZ LYRA, \\ JESSICA MENDES NADAL, PAULO VITOR FARAGO*
}

Laboratory of Drug Development and Industrial Pharmacy, Department of Pharmaceutical Sciences, State University of Ponta Grossa, Campus Uvaranas, Ponta Grossa, Parana, Brazil. Email: pvfarago@gmail.com

Received: 21 June 2020, Revised and Accepted: 29 July 2020

ABSTRACT

Objective: A simple and fast analytical method of ultra-high performance liquid chromatography (UHPLC) was developed and validated to quantify isradipine (ISR) in poly( $\varepsilon$-caprolactone)/polyethylene glycol nanocapsules.

Methods: Experiments were performed by UHPLC on a $\mathrm{C}_{18}$ chromatographic column at $25^{\circ} \mathrm{C}$, using a mobile phase composed by methanol and water $(85: 15 \mathrm{v} / \mathrm{v})$ with a flow rate of $0.5 \mathrm{ml} / \mathrm{min}$ and UV detection at $327 \mathrm{~nm}$ for achieving a total run time of $1.5 \mathrm{~min}$. The UHPLC method was validated according to the guidelines set on The International Conference on Harmonisation.

Results: It proved to be selective, linear ( $\mathrm{r}=0.99962)$, precise (relative standard deviation $<4.1 \%$ ), and accurate (recovery rates between $95.24 \%$ and $96.53 \%$ ) at the range from 10 to $40 \mu \mathrm{g} / \mathrm{ml}$. The performance was robust when slight changes in the flow rate, wavelength of detection, and mobile phase composition were tested. It was successfully applied to quantify ISR from nanoparticulate polymeric systems, showing a high loading efficiency rate, $>98.55 \%$.

Conclusion: These results provided an experimental basis to use this method for quantifying ISR with reliable results, besides being fast, easy to perform, and cheaper.

Keywords: Analytical method validation, Neuroprotective effect, Pharmaceutical quality control, Polymeric nanocapsules.

(C) 2020 The Authors. Published by Innovare Academic Sciences Pvt Ltd. This is an open access article under the CC BY license (http://creativecommons. org/licenses/by/4. 0/) DOI: http://dx.doi.org/10.22159/ajpcr.2020.v13i8.38817

\section{INTRODUCTION}

Quality control has been an essential tool for pharmaceutical industries since it ensures efficacy and safety for pharmaceutical products. Nevertheless, its results are only reliable when analytical methods employed are validated [1]. The validation of an analytical method is a major approach for assuring its quality, especially when the need to prove the quality of pharmaceutical analysis has been increasingly recognized and demanded since unreliable data can lead to wrong decisions and irrecoverable financial losses [2]. In this sense, ultra-high performance liquid chromatography (UHPLC) brought new opportunities in pharmaceutical industries to get faster analytical separation results while remaining the method quality. Its technique speed results in reduced consumption of solvent while presents a resolution and sensitivity greater than other conventional techniques [3].

The isradipine (ISR) or 3-methyl 5-propan-2-yl 4-(2,1,3-benzoxadiazol4-yl)-2,6-dimethyl-1,4-dihydropyridine-3,5-dicarboxylate is a calcium channel blocker which belongs to the dihydropyridine class and has demonstrated an interesting neuroprotective effect associated to its pharmacotherapeutic use [4]. In this case, the main challenge is to get this drug to cross the blood-brain barrier in therapeutic concentrations. The clinical study performed demonstrated that the dose of ISR as immediate-release formulation may have been insufficient to engage the target calcium channels associated with neuroprotective effects [5]. Thus, the research for strategies that make it possible to deflect the limitations related to the action of this drug in the central nervous system is growing and current.

In this context, polymeric nanocapsules have an interesting application for delivering drugs in the central nervous system, accompanied by its controlled release at the site [6,7]. Before the advantages offered by nanoparticulate systems, there is a demand for a reliable method capable of quantifying the drug in these formulations. Some chromatographic methods to quantify ISR have been previously reported in the literature [8-12]. However, none of them is appropriate to quantify drug-loaded polymeric nanocapsules, besides presenting complexes steps and wasting more expensive products. Hence, the aim of this paper was to develop and validate a reversed-phase UHPLCphotodiode array (RP-UHPLC-PDA) method to quantify ISR loaded in poly( $\varepsilon$-caprolactone) (PCL)/polyethylene glycol (PEG) nanocapsules.

\section{METHODS}

ISR hydrochloride (European Pharmacopoeia reference standard, CAS number 75695-93-1), PCL ( $M_{w}=10000 \mathrm{~g} / \mathrm{mol}$, Sigma-Aldrich, St. Louis, MO, USA), and PEG $\left(M_{w}=6000 \mathrm{~g} / \mathrm{mol}\right.$, Fluka, St. Louis, MO, USA) were used as received. Methanol of gradient grade for liquid chromatography was purchased from Merck (Darmstadt, Germany). All other chemicals used were of analytical grade.

\section{Preparation of polymeric nanocapsules containing ISR}

Nanocapsule suspensions obtained from PCL/PEG polymer blend (80:20) containing isradipine (NC-ISR) were prepared in triplicate by the interfacial deposition procedure of a preformed polymer developed and described by Fessi et al. [13], according to the formulation described in Table 1. Besides the loaded formulation, nanocapsules without the drug were also produced in triplicate as a negative control (NC-0). Briefly, the polymer blend was solvated in acetone, along with sorbitan monooleate, ISR, and caprylic/capric triglycerides to obtain the organic phase. Then, the organic phase was dripped slowly into the aqueous phase, previously prepared with ultrapure water and polysorbate 80 , and maintained under vigorous magnetic stirring and 
Table 1: Formulations of the loaded and non-loaded nanocapsule suspensions

\begin{tabular}{lllllllll}
\hline \multirow{2}{*}{ Formulation } & \multicolumn{2}{l}{ Organic phase } & & & Aqueous phase \\
\cline { 2 - 6 } & ISR (mg) & PCL (mg) & PEG (mg) & CCT (mg) & Acetone (ml) & Sorbitan monooleate (mg) & Polysorbate 80 (mg) & Water (m) \\
\hline NC-ISR & 20 & 80 & 20 & 300 & 27 & 77 & 77 \\
NC-0 & - & 80 & 20 & 300 & 27 & 77 & 77 \\
\hline
\end{tabular}

NC-ISR: Nanocapsules containing isradipine, NC-0: Negative control, PCL: Poly(E-caprolactone), PEG: Polyethylene glycol, CCT: Capric triglycerides

temperature between $40^{\circ} \mathrm{C}$ and $50^{\circ} \mathrm{C}$. In addition, the magnetic stirring was maintained for $10 \mathrm{~min}$ after the end of the drip and the solvent was eliminated by rotary evaporation at $50^{\circ} \mathrm{C}$, reaching a final volume of $10 \mathrm{ml}$ and a drug concentration of $2 \mathrm{mg} / \mathrm{ml}$.

In sequence, the resulting nanosuspension was added with $5 \%$ of sucrose $(\mathrm{w} / \mathrm{v})$, as a cryoprotective agent, frozen at $-80^{\circ} \mathrm{C}$ for $24 \mathrm{~h}$, and lyophilized in a Terroni ${ }^{\circledR}$ lyophilizer model LD1500 for $48 \mathrm{~h}$. To modify the surface of the nanocapsules [14], they were coated with polysorbate 80 after completing the conventional process for obtaining the nanoparticulate system. For this, the powder resulting from the freezedrying was resuspended in $2 \mathrm{ml}$ of a solution of polysorbate $80(1 \%$, $\mathrm{w} / \mathrm{v}$ ). The suspension remained incubated at room temperature for $30 \mathrm{~min}$, was frozen again at $-80^{\circ} \mathrm{C}$ for $24 \mathrm{~h}$, and freeze-dried for $72 \mathrm{~h}$. The final powder was stored in a desiccator at room temperature and protected from light.

\section{Chromatographic conditions}

Chromatographic conditions were tested and defined by the authors. Different composition, $\mathrm{pH}$, and flow rate of the mobile phase were tested, as well as column temperature and detection wavelength.

Experiments were performed in a Shimadzu Nexera System ${ }^{\circledR}$ UHPLC, equipped with a quaternary pump (LC-30AD), degasser (DGU-20A), automatic sampler (SIL-30 AC), thermostatic column compartment (CTO-20AC), and a photodiode array detector (SPD-M20A). The analytical column used was a Shimadzu Shim-pack XR-ODS III (C18; $2.0 \mathrm{~mm}$ internal diameter $\times 75 \mathrm{~mm}$ long; particle size of $1.6 \mu \mathrm{m})$. The isocratic mobile phase consisted of methanol:water $(85: 15 \mathrm{v} / \mathrm{v})$ with a flow rate of $0.5 \mathrm{ml} / \mathrm{min}$; temperature of $25^{\circ} \mathrm{C}$, injection volume of $10 \mu \mathrm{l}$, and wavelength detection at $327 \mathrm{~nm}$. The total analytical run time was $1.5 \mathrm{~min}$. The acquisition and processing of the data were obtained with the LabSolutions ${ }^{\circledR}$ Software (Shimadzu, Japan).

\section{Preparation of standard solutions}

A stock standard solution of $1 \mathrm{mg} / \mathrm{ml}$ of ISR was daily prepared by dissolving $10 \mathrm{mg}$ of the drug in a $10 \mathrm{ml}$ volumetric flask with methanol. This solution was further diluted in the mobile phase to prepare five different standard solutions ranging from 10 to $40 \mu \mathrm{g} / \mathrm{ml}$. Final solutions were filtered through a poly(vinylidene fluoride) membrane filter (Durapore ${ }^{\circledR}$ membrane filter, $0.22 \mu \mathrm{m}$ pore size) before injection into the UHPLC system.

\section{Preparation of sample solutions}

The final dosage form, a nanopowder, was resuspended in $10 \mathrm{ml}$ of purified water. The nanosuspension $(500 \mu \mathrm{l})$ was submitted to ultrafiltration in an Amicon ${ }^{\circledR}$ device $\left(M_{w}\right.$ cutoff $=10,000 \mathrm{~g} / \mathrm{mol}$, Merck Millipore) by centrifugation at $2200 \mathrm{rcf}$ for $30 \mathrm{~min}$. The amount of free ISR in ultrafiltration fraction was chromatographically quantified as proposed. All procedure was performed in triplicate.

\section{Method validation}

The UHPLC method was validated according to the guidelines set on The International Conference on Harmonization of Technical Requirements for Registration of Pharmaceuticals for Human Use [15]. Parameters evaluated were selectivity, linearity and range, limit of detection (LOD), limit of quantification (LOQ), precision, accuracy, and robustness.

Selectivity was investigated by comparing both chromatograms of the standard solution and the sample solution from $\mathrm{NC}-0$, obtained from the ultrafiltration of the nanosuspension prepared without ISR. Both solutions were chromatographed under previously defined conditions. The linearity of the method was assessed by preparing and analyzing of three analytical curves. For this, five concentration levels of ISR were obtained from dilutions of the standard solution in the mobile phase. The range was defined based on the intensity of the peaks produced in the chromatogram, including an interval from $10.0 \mu \mathrm{g} / \mathrm{ml}$ to $40.0 \mu \mathrm{g} / \mathrm{ml}$. The analysis of linear regression was performed using the least-squares method and the slope was tested by ANOVA at a significance level of 0.05. LOD and LOQ were obtained based on the standard deviation of the response $(s)$ and the slope (a) of three analytical curves (LOD = $3.3 \mathrm{~s} / \mathrm{a}$ and LOQ = $10 \mathrm{~s} / \mathrm{a}$ ). Repeatability and intermediate precision were carried out by assaying six different samples at the same concentration $(25.0 \mu \mathrm{g} / \mathrm{ml})$ under the same experimental conditions during the same day (morning, afternoon, and night) and along three different days with different analysts, respectively. These results were expressed as relative standard deviation (RSD). Accuracy was determined by the recovery test. Different standard solutions were prepared and added to a known quantity of ISR. Final solutions were analyzed in the chromatographic system in triplicate. Robustness of the method was assessed from variations of the recommended conditions (mobile phase composition, flow rate, and wavelength of drug detection).

\section{Method applicability: Determination of ISR loading efficiency}

The amount of ISR was calculated and reported as loading efficiency following the equation below. Loading efficiency (\%) was determined from the sample solution. Non-entrapped ISR was quantified in the ultrafiltrate by the proposed chromatographic method.

$$
\text { Loading efficiency } \%=\frac{\text { Total drug added }- \text { Non entrapped drug }}{\text { Total drug added }} \times 100
$$

\section{RESULTS}

\section{Preparation of polymeric nanocapsules containing ISR}

Nanocapsules from PCL/PEG polymer blend were successfully obtained by the proposed method. Formulations showed a powder aspect with a slightly yellowish-white coloring.

\section{Method validation}

The main parameters related to the proposed method for quantification of ISR are briefly described in Table 2 .

The UHPLC method presented a total run time of $1.5 \mathrm{~min}$ and proved to be selective since the NC-0 formulation did not show relevant signal detection overlap at the same reading position of the drug (Fig. 1).

The linearity test was performed at five different concentrations level of the analyte and its mean analytical curve obtained is shown in Fig. 2.

Values of angular coefficient (slope of the line), linear coefficient (Y-intercept), Pearson's correlation coefficient ( $r$ ), and coefficient of determination $\left(\mathrm{r}^{2}\right)$ were obtained based on the linear regression analysis and were able to evidence the quality of the linear regression obtained. Results are described in Table 3.

Results showed that the Pearson's correlation coefficient statistically is equal to 1 and a non-zero slope (significance level of $0.05 \%$ ).

To demonstrate that $y$ really varies as a function of $x$, it has been shown that the variation due to the regression (model) is sufficiently greater 
than that one due to the error (residuals). In this way, the residues were graphically plotted in Fig. 3.

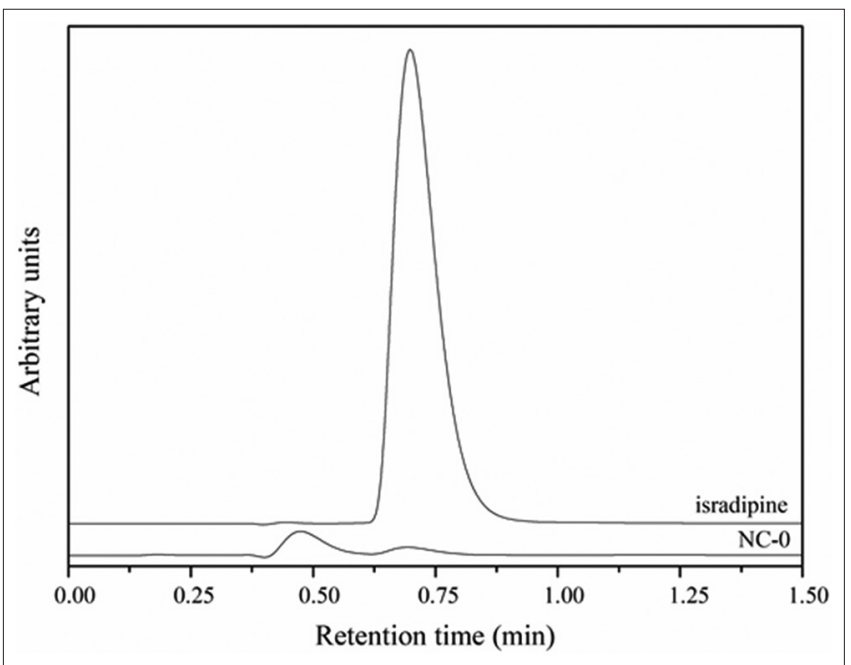

Fig. 1: Representative chromatogram of pure isradipine and NC-0 formulation

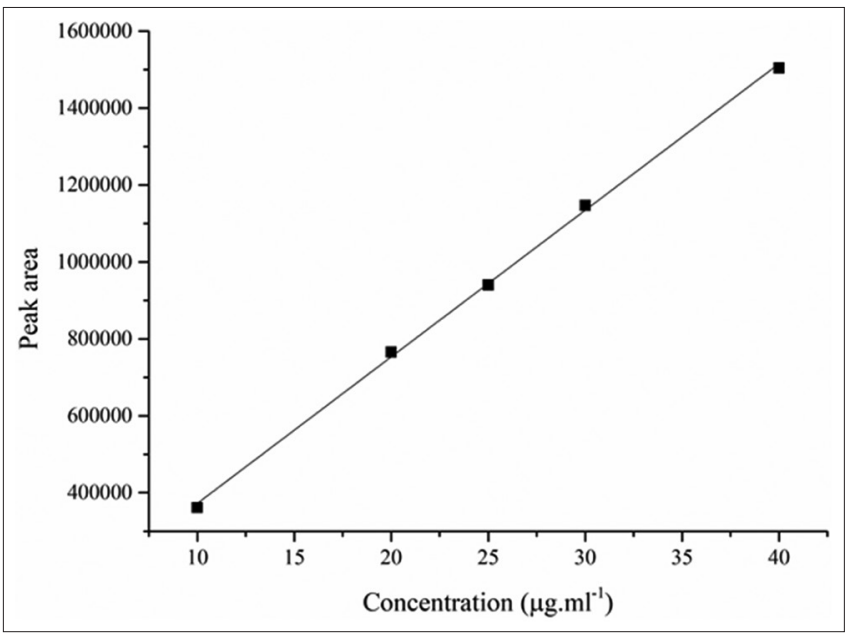

Fig. 2: Graphical representation of the mean analytical curve obtained to quantify isradipine by RP-UHPLC-PDA method at a concentration range from 10 to $40 \mu \mathrm{g} / \mathrm{ml}$

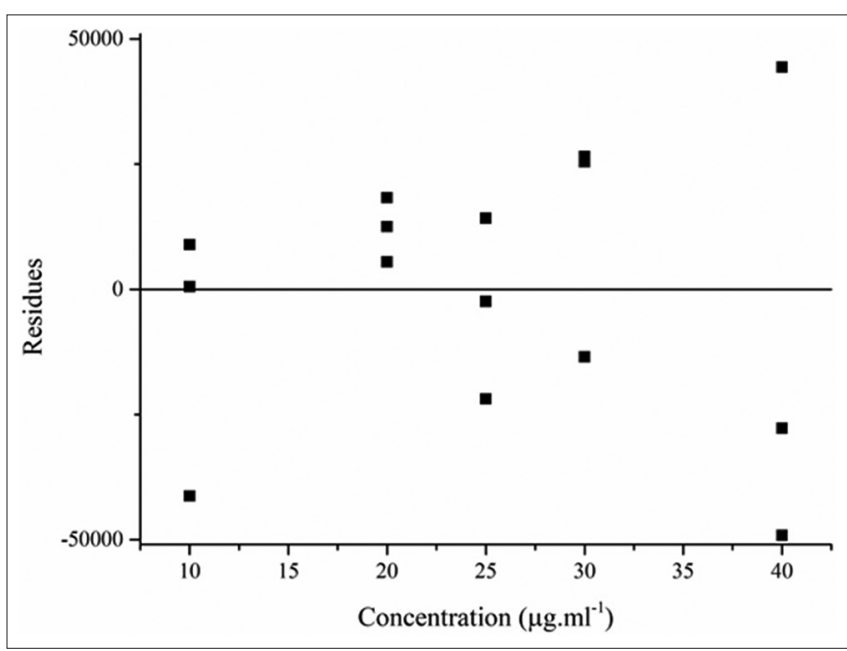

Fig. 3: Graphical representation of the residues obtained from replicates of each concentration level of the analytical curves
The visual analysis indicated that the residues are independent and homogeneously distributed according to the mean.

The developed method showed a LOD of $2.9 \mu \mathrm{g} / \mathrm{ml}$ and a LOQ of $8.78 \mu \mathrm{g} / \mathrm{ml}$.

The results of repeatability and intermediate precision obtained for this UHPLC-PDA method are shown in Table 4.

The obtained values of RSD were $<5.0 \%$.

Regarding accuracy, the recovery rate of ISR is between $95.24 \%$ and $96.53 \%$ for different concentration levels evaluated. Table 5 describes the obtained data.

Recovery rates obtained for robustness are summarized in Table 6 . These values showed a variation $<5 \%$ due to small changes in the flow rate, wavelength of detection, and the organic phase concentration in the mobile phase.

Method applicability: Determination of ISR loading efficiency Loading efficiency of ISR from nanocapsules was performed by the purposed RP-UHPLC-PDA method.

The chromatograms obtained from the ultrafiltrate samples showed no peaks; that is, there is no detectable concentration of free ISR in

Table 2: Main parameters of the RP-UHPLC-PDA method for quantifying isradipine from polymeric nanocapsules

\begin{tabular}{ll}
\hline Parameters & Results* \\
\hline Retention time (min) & $0.699 \pm 0.001$ \\
Width at 5\% of the peak height (min) & $0.208 \pm 0.001$ \\
Tailing factor & $1.485 \pm 0.006$
\end{tabular}

*Mean \pm standard deviation. RP-UHPLC-PDA: Reversed-phase-ultra-high performance liquid chromatography-photodiode array

Table 3: Linearity parameters of the RP-UHPLC-PDA method for quantifying isradipine from polymeric nanocapsules

\begin{tabular}{ll}
\hline Parameters & Results \\
\hline Linearity range & $10-40 \mu \mathrm{g} / \mathrm{ml}$ \\
Linear equation $(\mathrm{y}=\mathrm{ax}+\mathrm{b})$ & $\mathrm{y}=38100 \mathrm{x}-8723.8$ \\
Slope $(\mathrm{a})$ & 38100.0 \\
Y-intercept $(\mathrm{b})$ & -8723.8 \\
Pearson's correlation coefficient $(\mathrm{r})$ & 0.99962 \\
Coefficient of determination $\left(\mathrm{r}^{2}\right)$ & 0.99899 \\
\hline
\end{tabular}

RP-UHPLC-PDA: Reversed-phase-ultra-high performance liquid chromatography-photodiode array

Table 4: Results of precision assays of the RP-UHPLC-PDA method for quantifying isradipine from polymeric nanocapsules

\begin{tabular}{lll}
\hline Parameters & $\begin{array}{l}\text { Sample concentration } \\
(\mu \mathrm{g} / \mathrm{ml})\end{array}$ & $\mathrm{RSD}^{*}(\%)$
\end{tabular}

$\begin{array}{lll}\text { Repeatability }(\mathrm{n}=6) & 25 & 4.02 \\ \text { Intermediate } & 3.70\end{array}$

*RSD means relative standard deviation. RSD: Relative standard

deviation, RP-UHPLC-PDA: Reversed-phase-ultra-high performance liquid chromatography-photodiode array

Table 5: Results of accuracy assays of the RP-UHPLC-PDA method for quantifying isradipine from polymeric nanocapsules

\begin{tabular}{ll}
\hline $\begin{array}{l}\text { Theoretical sample concentration } \\
(\boldsymbol{\mu g} / \mathbf{m l})\end{array}$ & $\begin{array}{l}\text { Recovery rate }(\%) \\
(\mathbf{n}=3)\end{array}$ \\
\hline 20 & 95.95 \\
25 & 95.24 \\
30 & 96.53 \\
\hline
\end{tabular}

RP-UHPLC-PDA: Reversed-phase-ultra-high performance liquid chromatography-photodiode array 
Table 6: Results of robustness assays of the RP-UHPLC-PDA method for quantifying isradipine from polymeric nanocapsules

\begin{tabular}{|c|c|c|c|}
\hline Parameters & & $\begin{array}{l}\text { Theoretical sample concentration } \\
(\mu \mathrm{g} / \mathrm{ml})\end{array}$ & $\begin{array}{l}\text { Recovery rate }(\%) \\
(n=3)\end{array}$ \\
\hline \multirow[t]{2}{*}{ Flow rate $( \pm 0.02 \mathrm{ml} / \mathrm{min})$} & $0.48 \mathrm{ml} / \mathrm{min}$ & 25 & 104.21 \\
\hline & $0.52 \mathrm{ml} / \mathrm{min}$ & 25 & 96.21 \\
\hline \multirow{2}{*}{ Wavelength of detection $( \pm 2 \mathrm{~nm})$} & $325 \mathrm{~nm}$ & 25 & 99.98 \\
\hline & $329 \mathrm{~nm}$ & 25 & 99.94 \\
\hline \multirow[t]{2}{*}{ Mobile phase composition ( $\pm 2 \%$ of methanol) } & 83:17 (methanol:water) & 25 & 100.41 \\
\hline & $87: 13$ (methanol:water) & 25 & 100.78 \\
\hline
\end{tabular}

RP-UHPLC-PDA: Reversed-phase-ultra-high performance liquid chromatography-photodiode array

the nanosuspension. Thus, it is possible to assert that the prepared polymeric system was able to incorporate ISR in its interior, with efficiency $>98.55 \%$, considering the value that meets to the LOD of the method.

\section{DISCUSSION}

Pharmaceutical quality control requires that its results are reliable and reproducible. For this, it is imperative to use methods that meet these demands besides being fast, easy to perform, and inexpensive. In this sense, the method developed in this work is very fast and simple and presents a lower cost when compared to the other methods that have already been described for quantification of ISR, whereas the mobile phase is composed only by methanol and ultra-purified water [8-12].

Analytically, we can say that the peak width at the base is adequate to guarantee the quality of the developed method, on the other hand, the peak tailing factor shows that it is not perfectly symmetrical since the ideal symmetry is represented by values equal to 1 . However, Petersson et al. [16] describe that the UHPLC, which has columns with particle sizes smaller than $2 \mu \mathrm{m}$, produces tailing factor values significantly higher than the equivalent HPLC, which uses columns with $3 \mu \mathrm{m}$ particles when the same amount of sample is injected. Expressions have shown that the tailing factor values change with the particle size and the number of theoretical plates, and even so, UHPLC produces more efficient separations when considered adjacent peaks. Thus, the tailing factor value must be analyzed, not singly, but rather associated with the peak width values at the base, to obtain a better parameter for measuring the quality of the method.

The validation process is responsible for ensuring that the results obtained are befitting. Moreover, once the parameters recommended by official agencies are strictly respected, the validation is able to prove that the analytical method employed produces reliable results, and it is suitable for the purpose for which it was intended [15,17].

The method developed for quantification of ISR proved to be selective since no excipient of the formulation showed absorption of electromagnetic radiation at the selected wavelength [18]. In this way, it ensures the ability of the method to quantify the drug before any other component. It generates an exclusive response to ISR allowing ensuring a positive result for the analyte and negative for the other substances.

The linearity test showed the method ability to get replies proportionally equivalent to the concentration of the drug within the stated range. It is known that the closer to 1 the Pearson's correlation coefficient is, the smaller the dispersion among the points and the greater the safety of the results [19]. Thus, it is clear the relevance of using a calibration curve instead of a single point for standardization in the analysis routine. In addition, the visual analysis indicated that the residues are independent and homogeneously distributed according to the mean, ensuring the reliability of the reply generated by the method [20].

The LOD, which refers to the lowest concentration of the analyte that the method is able to detect, and the LOQ which is the smallest amount of substance that the method is able to quantify with precision and accuracy, confirmed the feasibility of applying the method within the stated concentration range [21].
Official agencies describe that precision must be able to assess the closeness among the values found in the experiments and must be expressed as repeatability and intermediate precision [15]. In this work, repeatability showed the agreement among the results found when the method was performed under the same conditions, in a short time interval. When the intermediate precision was tested, the method performance showed a low variation when the analysis were accomplished in different days and with different analyzers, at the same laboratory [22].

The results also showed the agreement between the data obtained against the actual reference value when employing the quantification method of ISR, proving to be accurate [23].

In addition, the method was evaluated for the ability to withstand slight variations in the conditions of analysis, without hazarding its accuracy. In view of the recovery values obtained, the method was considered robust. Its robustness values the results obtained from this method enforcement since we assume that it can be reproduced without major problems [21].

When the UHPLC method was assayed to quantify the ISR nanoencapsulated in polymeric nanocapsules, it results in a high encapsulation rate. This rate its completely expected due to the high lipophilicity of the drug (water solubility $<10 \mathrm{mg} / \mathrm{l}$ at $37^{\circ} \mathrm{C}$ ) [24], which optimizes its incorporation in the oily core of this nanosystem.

In summary, according to the presented results, the method was considered selective, linear for the proposed application range, precise, accurate, and robust; therefore, it is properly validated. In addition, it was successfully used for quantifying the ISR nanoencapsulated in polymeric nanocapsules, providing reliable results.

\section{CONCLUSION}

The RP-UHPLC-PDA analytical method proposed above showed to be selective, linear, precise, accurate, and robust at the tested range. It is suitable for determining ISR loaded in PCL/PEG nanocapsules. The obtained nanoformulation presented high loading efficiency. These results provided an experimental basis to use this method for quantifying ISR from polymeric nanosystems, providing reliable results, besides being fast, easy to perform, and cheaper.

\section{AUTHORS' CONTRIBUTIONS}

Conception and design: Fernanda Malaquias Barboza, Amanda Martinez Lyra, Paulo Vitor Farago.

Sample preparation: Fernanda Malaquias Barboza, Guilherme do Anjos Camargo.

Analytical method performance: Fernanda Malaquias Barboza, Guilherme do Anjos Camargo, Amanda Martinez Lyra.

Collection and assembly of data: Fernanda Malaquias Barboza, Guilherme do Anjos Camargo, Amanda Martinez Lyra.

Analysis and interpretation of the data: Fernanda Malaquias Barboza, Jessica Mendes Nadal. 
Drafting of the article: Fernanda Malaquias Barboza, Jessica Mendes Nadal, Paulo Vitor Farago.

Critical revision of the article for important intellectual content: Paulo Vitor Farago.

\section{CONFLICTS OF INTEREST}

The authors declare that there are no conflicts of interest.

\section{FUNDING}

This work was supported by the Conselho Nacional de Desenvolvimento Científico e Tecnológico - CNPq (grant number 311595/2016-2).

\section{REFERENCES}

1. Lachman L, Lieberman HA, Kaning JL. Teoria e Prática na Indústria Farmacêutica. Lisboa: Fundação Calouste; 2001.

2. La Roca MF, Sobrinho JL, Nunes LC, Neto PJ. Desenvolvimento e validação de método analítico: Passo importante na produção de medicamentos. Rev Bras Farm 2007;88:177-80.

3. Kumar A, Saini G, Nair A, Sharma R. UPLC: A preeminent technique in pharmaceutical analysis. Acta Pol Pharm 2012;69:371-80.

4. Chan CS, Guzman, JN, Ilijic E, Mercer JN, Rick RC, Tkatch T, et al. Rejuvenation protects neurons in mouse models of Parkinson's disease. Nature 2007;447:10819.

5. Parkinson Study Group STEADY-PD III Investigators. Isradipine versus placebo in early Parkinson disease: A randomized trial. Ann Intern Med 2020;172:591-8.

6. Singh R, Lillard JW Jr. Nanoparticle-based targeted drug delivery. Exp Mol Pathol 2009;86:215-23.

7. Deng S, Gigliobianco MR, Censi R, Di Martino P. Polymeric nanocapsules as nanotechnological alternative for drug delivery system: Current status, challenges and opportunities. Nanomaterials (Basel) 2020;10:847-83.

8. Yang X, Xu H, Gan J, Li L, Li J, Jin Y, et al. Quantitative determination of isradipine in dog plasma by an ultra-performance liquid chromatography-tandem mass spectrometry method. Asian J Pharm Sci 2013;8:312-3

9. Aswini GL, Dachinamoorty D, Babu YR, Surekha ML, Swamy GK. Development and validation of isradipine in bulk and in its pharmaceutical formulation by RP-HPLC method. Int Res J Pharm 2012;3:131-3.

10. Chaitanya KK, Sankar DG, Israel DS, Lakshmi AV, Kumar CH. Isocratic-reverse phase liquid chromatographic method for the quantification of isradipine by UV detection in tablets. Eur J Appl Eng Sci Res 2012;1:220-7.

11. Rao RN, Nagaru P, Srinivasulu C, Krishnamurthy PR, Sireesha D, Bhaskar SU. Reverse phase HPLC method for the estimation of isradipine in pharmaceutical dosage forms. Asian J Chem 2005;17:1406-10.

12. Al-Suwayeh SA. Quick, simple, and sensitive HPLC method for determination of isradipine in plasma and its application in pharmacokinetic studies. Anal Lett 2002;35:1205-13.

13. Fessi H, Puisieux F, Devissaguet JP, Ammoury N, Benita S. Nanocapsule formation by interfacial polymer deposition following solvent displacement. Int J Pharm 1989;55:1-4.

14. Gelperina S, Maksimenko O, Khalansky A, Vanchugova L, Shipulo E, Abbasova $\mathrm{K}$, et al. Drug delivery to the brain using surfactant-coated poly (lactide-co-glycolide) nanoparticles: Influence of the formulation parameters. Eur J Pharm Biopharm 2010;74:157-63.

15. The International Conference on Harmonisation of Technical Requirements for Registration of Pharmaceuticals for Human Use. Validation of Analytical Procedures: Text and Methodology Q2 (R1); 2005.

16. Petersson P, Forssenb P, Edstrome L, Samie F, Tattertone S, Clarke A, et al. Why ultra-high performance liquid chromatography produces more tailing peaks than high performance liquid chromatography, why it does not matter and how it can be addressed. J Chromatogr A 2011;1218:6914-21.

17. Nadal JM, Toledo MG, Pupo YM, de Paula JP, Farago PV, Zanin SM. A stability-indicating HPLC-DAD method for determination of ferulic acid into microparticles: Development, validation, forced degradation, and encapsulation efficiency. J Anal Methods Chem 2015;2015:286812.

18. Gomes ML, Klein T, Simionatto M, Nadal JM, Zanin SM, Borsato DM, et al. A simple RP-HPLC/UV method for determination of cilostazol in polymeric nanoparticles suspensions: Development and validation. Acta Pharm 2015;34:803-9.

19. Almeida MA, Nadal JM, Klein T, de Paula JP, Budel, JM, Novatiski A, et al. Innovative phytoformulation containing capsaicinoids: Microparticles development, analytical method validation, and antiulcer effect. Pharmacogn Mag 2018;14:290-6.

20. Gaspar MD, Lyra AM, Camargo GA, Nadal JM, Teitelbaum AP, dos Santos FA, et al. Development and validation of a reversed-phase UHPLC-PDA method for determination of chlorhexidine in meth (acrylic) nanocapsules. Lat Am J Pharm 2020;39:792-8.

21. Lyra AM, Nadal JM, Klein T, Farago PV. Development and validation of a simple RP-HPLC method for determination of efavirenz in polymeric microparticles. Lat Am J Pharm 2017;36:2354-62.

22. Lopes CE, Langoski G, Klein T, Ferrari PC, Farago PV. A simple HPLC method for the determination of halcinonide in lipid nanoparticles: Development, validation, encapsulation efficiency, and in vitro drug permeation. Braz J Pharm Sci 2017;53:1-9.

23. Cartagena-Molina AF, Klein T, Lyra AM, Urban AM, Farago PV, Campanha NH. Development and validation of an RP-HPLC/ UV method for determination of miconazole nitrate in spray-dried polymeric microparticles. Acta Farm Bonaer 2016;35:1354-60.

24. Ramasahayam B, Eedara BB, Kandadi P, Jukanti R, Bandari S. Development of isradipine loaded self-nano emulsifying powders for improved oral delivery: In vitro and in vivo evaluation. Drug Dev Ind Pharm 2015;41:753-63. 